\title{
Segmentation of the Liver from Abdominal CT Using Markov Random Field model and GVF Snakes
}

\author{
Raja S Alomari, Suryaprakash Kompalli, Vipin Chaudhary \\ The Department of Computer Science and Engineering, \\ University at Buffalo, The State University of New York, Buffalo, NY
}

\begin{abstract}
Liver segmentation from scans of the abdominal area is an important step in several diagnostic processes. $C T$ scans of the abdominal area contain several organs in close proximity exhibiting similar image characteristics. In this paper, we present preliminary results on an algorithm that uses Markov Random Fields to obtain an initial contour of the liver. Gradient vector fields (GVF) and active contours are used to refine the initial estimate and segment the liver. Tests are reported on 13 clinical cases using a similarity metric that combines area and space.
\end{abstract}

\section{Introduction}

Segmentation of the liver from images of the abdominal area is a critical first stage in diagnostic and surgical procedures. Several organs such as stomach, kidney, and heart are adjacent to the liver and exhibit similar material characteristics in CT images. This similarity creates weak boundaries and partial-volume effects that make it difficult to discriminate the liver from adjacent tissues. In addition these are critical life-supporting organs and techniques that wrongly segment them as part of the liver would be unacceptable. Differences in liver shape, size, and other features between patients adds further complexity to the segmentation problem.

Liver segmentation methodologies include modeldriven approaches that use a pre-computed model in addition to the input image $[11,5,8,9]$, and datadriven approaches that apply processing techniques on only the input image $[14,17]$. There are also hybrid techniques $[16,10]$ that analyze the CT data to obtain an approximate estimate of the liver region, and enhance the estimates by using model-driven approach. Our methodology falls into the latter case, where we use a Markov Random Field to obtain an initial liver boundary and refine the boundary using an Active Contour.

Hybrid techniques are often targeted at reducing the amount of manual intervention needed in liver segmentation. However, complexity of the task and critical nature of errors makes a completely automated technique very elusive. For example, Liu et al $[10,2]$ evaluate the histograms of a contrast-enhanced CT slice to obtain thresholds that serve as a band-pass filter that can highlight the liver. The band-pass is used as a mask that can provide an initialization of the GVF snake. These histogram techniques examine image properties taken globally and fail when the liver occupies small percentage of the abdomen. We model the image as Markov Random Field (MRF) to accommodate both the local and global distribution of CT image values and automatically label pixels of the image that correspond to the liver. The initial boundary provided by MRF modeling is enhanced by using GVF active contours. In a different approach, MRF modeling has been used to obtain texture features from Dicom pixel values [15]. A GVF snake is designed to use the the texture features and output the liver boundary.

Chen et. al. [5] extract normalized fractional brownian features from a CT image, and apply a Neural network classifier to obtain an initial estimate of the liver region. The initial estimate is refined using GVF snakes. This approach has the potential to work effectively in slices where the liver area is limited. However, the approach is targeted at designing classifiers that can identify different types of liver cancers, and does not evaluate effectiveness of the liver boundary segmentation itself. More recent work by Soler et. al. [18] uses thresholding parameters to perform initial segmentation, then applies geometrical constraints that encode anatomical information of the liver structure. The authors report effectiveness of the visualization system for surgery, but the results have not been analyzed in light of segmentation error metrics. In this work, we discuss standard error metrics that have been reported 
for liver segmentation and propose a new metric that takes into consideration the spatial distribution of segmentation errors.

\section{Methodology}

CT scans of the abdominal area are available in DICOM format, with a slice thickness of $5 \mathrm{~mm}$. Each slice has size of 512 X 512, and each pixel contains a 12 bit HU (Hounsfield unit, Equation 1) value. The Slope and Intercept values refer to the Rescale Slope and Intercept obtained from the DICOM header. HU values are based on the physical properties of tissues and it is known that the liver has a $\mathrm{HU}$ value in between 50 and $250[7,13]$. We obtain a gray scale image from the input DICOM file by mapping the HU units between $50-250$ to $1-254$ (Figure 1). HU units outside the range of 50-250 are mapped to 0 or 255 .

$$
\begin{aligned}
H U & =\text { DICOMvalue } * \text { Slope }+ \text { Intercept } \\
x & =\left\{\begin{array}{cc}
0 & \text { if } \mathrm{HU}<50 \\
255 & \text { if } \mathrm{HU}>250 \\
H U * 254 / 200 & \text { Otherwise }
\end{array}\right.
\end{aligned}
$$

\subsection{Markov Random Fields for Initial Estimate}

Markov Random Field is a popular statistical technique in which the property associated with each image pixel is conditioned on its immediate neighborhood. An image $S$ is said to be an MRF if: $\forall s \in$ $S, p\left(Y_{s} \mid Y_{r}, r \neq s\right)=p\left(Y_{s} \mid Y_{\delta_{s}}\right)$, where $s$ and $r$ are pixels on the image, and $\delta_{s}$ is a neighborhood of $s$. Researchers model an MRF by taking $Y$ as a specific property of the image, say a class label assignment, or in cases like [15], $Y$ can be a feature of the image.

We have implemented the Modified Metropolis Dynamics [3] (MMD) to model $Y$ as class label assignment for CT slices of the abdominal area. From Baye's theorem, we have: $P(Y \mid X)=P(X \mid Y) P(Y) / P(X)$. The numerator can be ignored as a normalizing factor, and we will have:

$$
P(Y \mid X) \propto P(X \mid Y) P(Y)=\prod_{i=1}^{N} P\left(x_{i} \mid y_{i}\right) \prod_{c \in \zeta} \exp \left(-\Psi_{c Y}\right)
$$
where $\zeta$ is the set of cliques

The first product on the RHS of Equation 2 represents the class label assignment based on pixel property and is modeled as a gaussian over the grayscale value: $p\left(x \mid y_{i}\right)=1 / \sigma_{i} \sqrt{2 \pi} \exp \left\{\left(x-\mu_{i}\right)^{2} / 2 \sigma_{i}^{2}\right\}$, where $i$ is the corresponding class label. The second term on

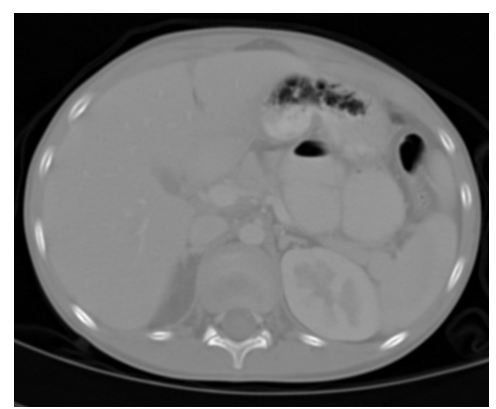

(a) Image without the use of window/level setting

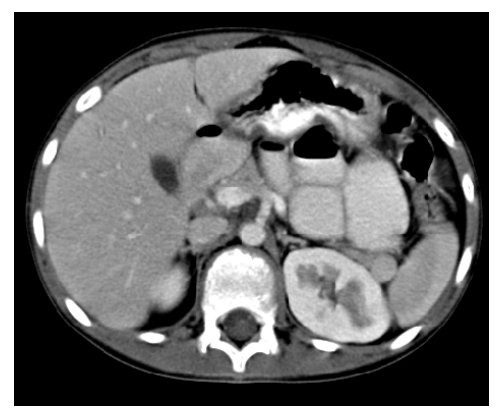

(b) Mapping $\mathrm{Hu}$ values to grayscale with a window/level setting of 150/100

\section{Figure 1. Using window/level settings to map Hounsfield unit values to grayscale values}

RHS follows from the MRF definition. It is defined as follows:

$$
\begin{aligned}
P(Y) & =\sum_{\left\{x_{i}, x_{j}\right\} \in \zeta} \Delta\left(Y_{x_{i}}, Y_{x_{j}}\right) \\
\Delta\left(Y_{x_{i}}, Y_{x_{j}}\right) & =\left\{\begin{array}{cl}
4 * \beta & Y_{x_{i}} \neq Y_{x_{j}} \\
-1 * \beta & \text { otherwise }
\end{array}\right.
\end{aligned}
$$

The CT slice is assumed to be composed of three classes, air, bone and soft tissue. The mean and variance $\left(\mu_{i}, \sigma_{i}\right)$ are updated using the class labels at each MMD iteration. Statistics for the first iteration are obtained using a 10x10 neighborhood around three initial seed points. The parameter $\beta$ which determines clique potential is empirically selected.

For each CT slice of the abdominal area, the user is presented with an image having grayscale values as per equation 1 . Histogram analysis is used to automatically select one pixel corresponding to air (The darkest blob in the image), and one pixel corresponding to bone (Brightest blob in the image). The user selects one seed pixel on the liver. Using these three seed points, the system calculates an initial value for the mean and 
variance of the respective classes $\left(\mu_{i}, \sigma_{i}\right)$. The MMD algorithm is now initiated by randomly assigning labels to each pixel in the image. At each iteration, the label at each pixel is updated using equation 2 . The algorithm terminates when we observe no change in the labels assigned to a pre-determined number of pixels (97\% of the image). The result of MMD is an approximate contour of the liver which we further enhance using active contours.

\subsection{Active Contours for Refinement}

Active contours have been widely used for liver segmentation, for example Liu et al. [10, 11] which uses gradient vector flow (GVF) field as an external force. Active contours are obtained by defining curves whose shape and location is guided by two forces: internal forces that are derived from pixels on the curve to impose desirable properties on the curve shape and external forces that are derived from image characteristics to limit the curve to certain locations in the image.

We employed the canny edge detector to derive an edge map that best follows the liver contour. The edge map is used to compute a gradient vector field which serves as the force field for energy minimization of the initial contour obtained from the MRF/MMD processing. This initial contour is then refined by the GVF snake to produce the final contour that minimizes the energy level. Elasticity, rigidity, viscosity, and external force weight have been set to $0.6,0.01,1$, and 0.6 respectively. Figure 2 shows an example of the GVF snake segmentation and manual ground truth.

\section{Evaluation of Liver Segmentation}

The algorithm is being evaluated using a dataset that will be made publicly available [1]. For every case, we have collected two sets of annotations from $4 \mathrm{hu}-$ man subjects, one radiologist and three students. Two annotations of the same case by an individual is separated by at least 24 hours. Each student annotation is rated by the radiologist using a 5-level scale ranging from accurate (1) to unacceptable (5). Thirteen cases have been collected so far, and the average rating for the three students is 1.29 .

Metrics used to estimate the accuracy of liver segmentation have included accuracy and error [11] Accuracy $=(R \cap G) / G$, Error $=(R \cup G-R \cap G) / G$ where $R$ and $G$ are areas of the automated and the gold standard segmentation results respectively. Pohle and Toennies [17] use four metrics for segmentation evaluation: Average deviation from the contour, Modified

\begin{tabular}{|c|c|c|}
\hline \multirow{2}{*}{ Case } & \multicolumn{2}{|c|}{ Error $=(R \cup G-R \cap G) / G[11]$} \\
\cline { 2 - 3 } & Mean $(\mu)$ & Variance $\left(\sigma^{2}\right)$ \\
\hline 1 & 0.1345 & 0.0180 \\
2 & 0.1518 & 0.0196 \\
3 & 0.2199 & 0.1153 \\
4 & 0.2482 & 0.1631 \\
5 & 0.1997 & 0.0847 \\
6 & 0.1654 & 0.0550 \\
7 & 0.3331 & 2.8066 \\
8 & 0.3537 & 2.8101 \\
9 & 0.3864 & 2.8644 \\
10 & 0.2998 & 2.7799 \\
11 & 0.2997 & 2.7779 \\
12 & 0.3183 & 2.7842 \\
13 & 0.3009 & 2.7851 \\
\hline Avg: & 0.2624 & 1.5433 \\
\hline
\end{tabular}

Table 1. Mean and variance of the error measure for GVF snake segmentation.

Hausdorff distance [4], Number of oversegmented pixels, and Number of undersegmented pixels. Some researchers have also used volumetric measures and ROC analysis $[16,19,6,12]$ to compute segmentation accuracy.

We introduce an error metric that is based on modified Hausdorff distance and takes into account the spatial distribution of erroneous pixels. It is reasonable to penalize over-segmentation errors that are spatially distant from the liver in comparison to over-segmentation errors that occur closer to the liver surface. We utilize area and modified Hausdorff distance metric (Equation 4) to quantify the similarity between two segmentation results.

$$
\begin{array}{ll}
\phi(A, B) & =\sum_{i=1}^{|A|} \min \left[\left\|a_{i}, B\right\|\right] \\
d_{\text {extra }} & =\phi(R-(R \cap G), G) \\
d_{\text {less }} & =\phi(G-(R \cap G), G)
\end{array}
$$

Where $A$ and $B$ are convex regions and $a_{i} \in A,\left\|a_{i}, B\right\|$ is distance of pixel $a_{i}$ from surface of $B, R$ is the segmented region or student annotation and $G$ is the expert annotation

Empirical evaluation conducted by overlaying expert annotations with results from the GVF snake shows strong similarity between the two (Figure 2). 


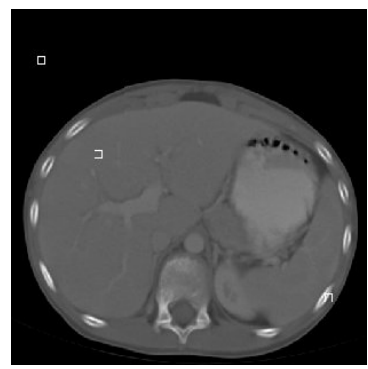

(a) Selection of seed points

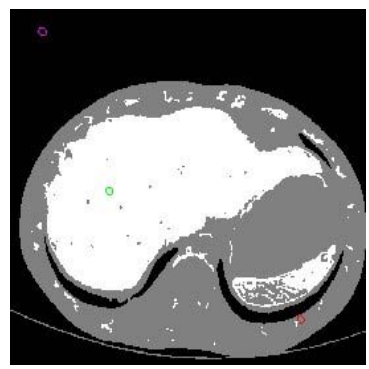

(b) MRF Result

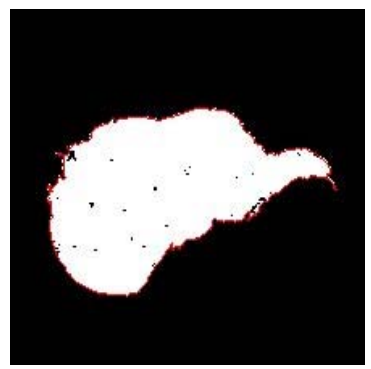

(c) Selecting largest MRF blob

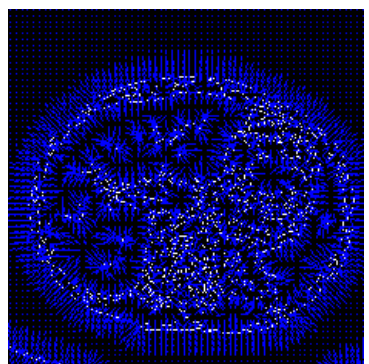

(d) Gradient Vector Field

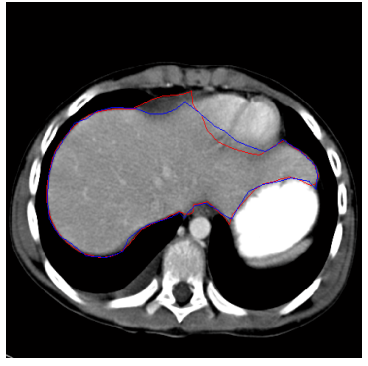

(e) Segmentation result

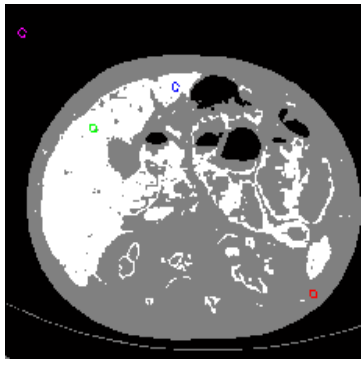

(f) MRF Result

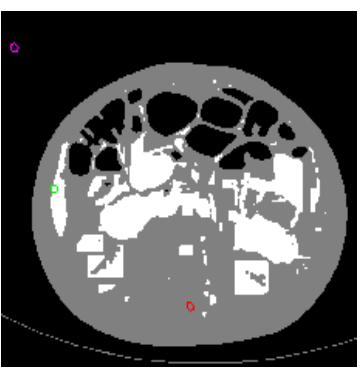

(i) MRF Result

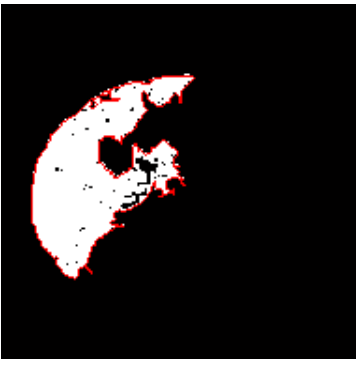

(g) Selecting largest MRF blob

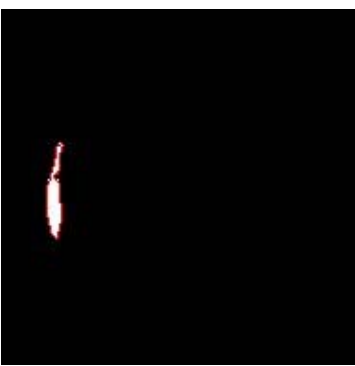

(j) Selecting largest MRF blob

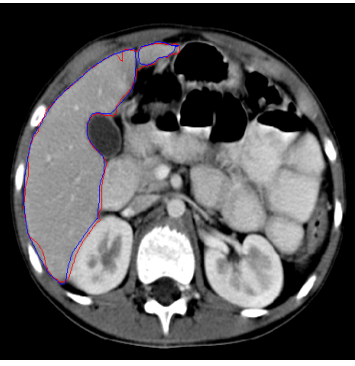

(h) Segmentation result

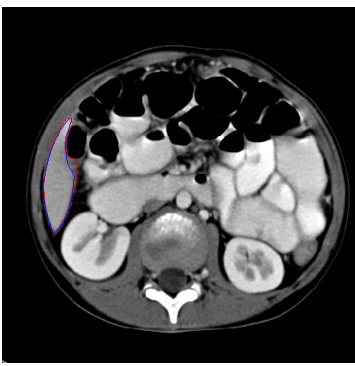

(k) Segmentation result

Figure 2. Using Markov Random Fields and GVF Snakes to segment liver 


\section{Discussion}

We present a new approach to liver segmentation that combines Markov Random Fields and Gradient Vector Field snakes to provide segmentation of the liver tissue. The only input required from the user is a single pixel inside the liver. While any error in the segmentation of medical organs is harmful, the limitations of imaging leads to partial volume effects, organ overlap, and other artifacts in image signals. Such aberrations can inherently cause variation among expert manual annotations, and possibly lead to errors in automatic segmentation algorithms. We are working on collecting a larger dataset and performing a comparison of our automatic segmentation technique with inter and intra user variation.

In automatic segmentation, it is likely to have errors that are spatially removed from the liver surface. It is reasonable to consider that such errors are a result of more gross failure and should be penalized more than errors that occur closer to the liver surface. We will contrast our metric that accounts for spatial distances in error with previously reported techniques like Accuracy and Error.

\section{Acknowledgments}

This research has been funded in part by New York State Office of Science, Technology and Academic Research. We would like to thank Dr. Stanley Lau from the Women and Children Hospital, Buffalo, NY for providing the datasets, and Yaqub Mohammed for assisting in data collection.

\section{References}

[1] Raja S Alomari, Surya Kompalli, Stanley T Lau, and Vipin Chaudhary. Design of a benchmark dataset, similarity metrics, and tools for liver segmentation. In Proceedings of the SPIE Medical Imaging Conference (To Appear), 2008.

[2] Kyongtae T. Bae, Maryellen L. Giger, Chin-Tu Chen, and Jr. Charles E. Kahn. Automatic segmentation of liver structure in ct images. Medical Physics, 20(1):71-78, 1993.

[3] Mark Berthod, Zoltan Kato, Shan Yu, and Josiane Zerubia. Bayesian image classification using markov random fields. Image and Vision Computing, 14:285-295, 1996.
[4] Kim Y Chalana V. A methodology for evaluation of boundary detection algorithms on medical images. IEEE Trans. on Medical Imaging, 16(5):642652, 1997.

[5] E. Chen, P. Chung, C. Chen, H. Tsai, and C. Chang. An automatic diagnostic system for CT liver image classification. IEEE Transactions on Biomedical Engineering, 45:783-794, 1998.

[6] Charles Florin, Nikos Paragios, Gareth FunkaLea, and James Williams. Liver segmentation using sparse $3 \mathrm{~d}$ prior models with optimal data support. In Proceedings of the IPMI, pages $38-49$, 2007.

[7] G. N. Hounsfield. Computed Medical Imaging. Science, 210:22-28, oct 1980.

[8] Shaohui Huang, Boliang Wang, and Xiaoyang Huang. Using gvf snake to segment liver from ct images. In Proceedings of 3rd IEEE/EMBS International Summer School on Medical Devices and Biosensors, 2006, pages 145-148, 2006.

[9] C. Krishnamurthy, J.J. Rodriguez, and R.J. Gillies. Snake-based liver lesion segmentation. In Southwest04, pages 187-191, 2004.

[10] F. Liu, B. Zhao, P. Kijewski, M. S. Ginsberg, L. Wang, and L. H. Schwartz. Automatic liver contour segmentation using GVF snake. In J. M. Fitzpatrick and M. Sonka, editors, Medical Imaging 2004: Image Processing. Edited by Fitzpatrick, J. Michael; Sonka, Milan. Proceedings of the SPIE, Volume 5370, pp. 1466-1473 (2004)., pages 14661473, May 2004.

[11] F. Liu, B. Zhao, P. K. Kijewski, L. Wang, and L. H. Schwartz. Liver segmentation for ct images using gvf snake. Medical Physics, 32(12):36993706, December 2005.

[12] Pham M., Susomboon R., Disney T., Raicu D., and Furst J. A comparison of texture models for automatic liver segmentation. In Medical Imaging 200\%: Image Processing. Edited by Pluim, Josien P. W.; Reinhardt, Joseph M.. Proceedings of the SPIE, Volume 6512, pp. 65124E (2007)., volume 6512 of Presented at the Society of PhotoOptical Instrumentation Engineers (SPIE) Conference, mar 2007.

[13] William W. Mayo-Smith, Himanshu Gupta, Mark S. Ridlen, Jeffrey M. Brody, Nancy C. Clements, and John J. Cronan. Detecting Hepatic 
Lesions: The Added Utility of CT Liver Window Settings. Radiology, 210(3):601-604, 1999.

[14] Yoshiharu Nakayama, Qiang Li, Shigehiko Katsuragawa, Ryuji Ikeda, Yasuhiro Hiai, Kazuo Awai, Shinichiro Kusunoki, Yasuyuki Yamashita, Hideaki Okajima, Yukihiro Inomata, and Kunio Doi. Automated hepatic volumetry for living related liver transplantation at multisection ct 1 . $R a$ diology, 240(3), September 2006.

[15] Carl Philips, Ruchaneewan Susomboon, Reem Mokhtar, Daniela Raicu, and Jacob Furst. Segmentation of soft tissue using texture features and gradient snakes. Technical Report TR07-011, CTI DePaul, 2007.

[16] R. Pohle, T. Behlau, and K. D. Toennies. Segmentation of 3D medical image data sets with a combination of region-based initial segmentation and active surfaces. In M. Sonka and J. M. Fitzpatrick, editors, Medical Imaging 2003: Image Processing. Edited by Sonka, Milan; Fitzpatrick, J. Michael. Proceedings of the SPIE, Volume 5032, pp. 12251232 (2003)., pages 1225-1232, may 2003.

[17] Klaus D. Toennies Regina Pohle. A new approach for model-based adaptive region growing in medical image analysis. In CAIP '01: Proceedings of the 9th International Conference on Computer Analysis of Images and Patterns, pages 238-246, Otto-von-Guericke University Magdeburg,, Department of Simulation and Graphics, London, UK, Regina@isg.cs.unimagdeburg.de,Klaus@isg.cs.uni-magdeburg.de, 2001. Springer-Verlag.

[18] L. Soler, H. Delingette, G. Malandain, J. Montagnat, N. Ayache, J.-M. Clement, C. Koehl, O. Dourthe, D. Mutter, and J. Marescaux. Fully automatic anatomical, pathological, and functional segmentation from ct scans for hepatic surgery. In K. M. Hanson, editor, Proc. SPIE Vol. 3979, p. 246-255, Medical Imaging 2000: Image Processing, Kenneth M. Hanson; Ed., pages 246 255 , jun 2000.

[19] Heimann T, Wolf I, and Meinzer HP. Active shape models for a fully automated $3 \mathrm{~d}$ segmentation of the liver - an evaluation on clinical data. In Proceedings of the MICCAI, pages $41-48$. Springer, 2006. 